Gefässchirurgie 2009 · 14:79-79

DOI 10.1007/s00772-009-0683-3

(c) Springer Medizin Verlag 2009

\author{
D. Böckler \\ Klinik für Gefäßchirurgie, Vasculäre und Endovasculäre Chirurgie \\ Universitätsklinikum Heidelberg
}

\title{
Der Aortenbogen
}

\section{- die therapeutische Herausforderung der nächsten Jahre!}

Mittlerweile sind 14 Jahre seit der ersten Publikation zur endovaskulären Versorgung thorakaler Aneurysmen durch die Stanford-Arbeitsgruppe um Michael Dake vergangen [1]. Darin wurden Patienten mit, aus heutiger Sicht, ,einfachen“ Aneurysmen der Aorta descendens im geraden Abschnitt. Nienaber et al. publizierten fünf Jahre später ebenfalls im NEJM eine vergleichbar kleine randomisierte Studie, bei der Patienten mit Aortendissektion Typ Stanford B entweder offen oder endovaskulär versorgt wurden [2]. Beide Studien sind sicherlich als die Sternstunde der thorakalen endovaskulären Aortenrekonstruktion (TEVAR) zu betrachten, auch wenn einer der Schwachpunkte die kleine Fallzahl in beiden Studien bei elektiver Indikationsstellung darstellt. Seither wurden keine weiteren prospektiv randomisierten Vergleichsstudien zur offenen und endovaskulären Therapie thorakaler Aortenpathologien durchgeführt bzw. veröffentlicht. Die geringere Mortalität und Morbidität, insbesondere die Paraplegierate, lassen dies wohl auch in Zukunft ethisch nicht mehr vertretbar erscheinen und führten dazu, dass TEVAR zur Therapie der ersten Wahl in vielen Zentren wurde. Metaanalysen und Registerdaten stellen somit zukünftig den höchsten Evidenzlevel dar.

Ohne die Bedeutung dieser eingangs zitierten publikatorischen Meilensteine schmälern zu wollen, spiegeln sie, damals wie heute, nicht zwangsläufig den klinischen Alltag wieder. Dies liegt zum einen daran, dass eine große Anzahl der thorakalen Stentgraft-Implantationen unter Notfallbedingungen durchgeführt werden und zum anderen, und das ist entscheidend, die Mehrzahl aller thorakalen Aortenpathologien nicht alleinig in der pars descendens der Aorta thoracica liegt, sondern eine Beteiligung des Aortenbogens in unmittelbarer Nachbarschaft zu den supraaortalen Äste aufweist. Auch wenn aktuell ein umfassendes interdisziplinär ausgearbeitetes Konsensuspapier verfasst wurde [3], befindet sich TEVAR insbesondere im Aortenbogen in einem ständigen, schnellen Fluss und ist als „work in process“ zu verstehen.

In den beiden folgenden Ausgaben unserer Fachzeitschrift werden Übersichtsarbeiten zur Epidemiologie und Ätiologie von Aortenbogen-Pathologien, aber auch deren moderne Schnittbildgebung diskutiert.

Letztere wiederum ist zwingende Voraussetzung für Indikationsstellung, Patientenselektion sowie Planung und Verfahrenswahl. Zur Therapie stehen uns mittlerweile neben dem konventionellen Bogenersatz (mit cerebraler Perfusion), Hybridoperationen und komplett endovaskuläre Ausschaltung mit Fenestrierungen bzw. side-branched Technologie sowie die chimney graft - Technik zu Verfügung. Alle Optionen werden in diesen beiden Themenheften vorgestellt und in ihrer Wertigkeit kritisch beleuchtet werden. Am Beispiel des traumatischen Aortenabrisses wird das wichtige Thema der mechanischen Beanspruchung von Stentgrafts analysiert.

Besonders freut mich, dass Tim Chuter aus San Francisco, der endovaskuläre Pionier auf dem Gebiet der side-branched Technologie, über seine mittlerweile 5-jährige persönliche Erfahrung und seine Zukunftsvisionen zur endovaskulären Therapie des Aortenbogens berichtet.

Spätestens nach der Lektüre beider Hefte dürfte hoffentlich jeder Leser erkennen, vor welch großer therapeutischen Herausforderung im Aortenbogen wir Gefäßmediziner und im speziellen wir Chirurgen in den kommenden Jahre stehen.

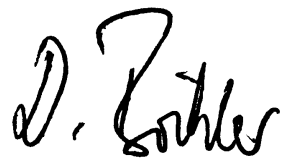

Prof. Dr. D. Böckler

\section{Korrespondenzadresse Prof. Dr. D. Böckler

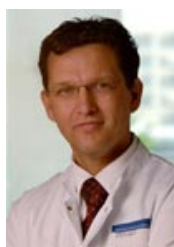 Klinik für Gefäßchirurgie Vasculäre und Endo- vasculäre Chirurgie Universitätsklinikum Heidelberg Im Neuenheimer Feld 110 69120 Heidelberg dittmar.boeckler@med. uni-heidelberg.de}

\section{Literatur:}

1. Dake MD, Miller C, Semba C, al. e. Transluminal placement of endovascular stentgrafts for the treatment of descending thoracic aortic aneurysms. N Engl J Med 1994; 331:1729-1734.

2. Nienaber CA, Fattori R, Lund $G$, et al. Nonsurgical reconstruction of thoracic aortic dissection by stent-graft placement. $\mathrm{N}$ Engl J Med. 1999;340:1539-1545

3. Svennson LG, Kouchoukos NT, Miller DC et al. Expert consensus document on the treatment of descending thoracic aortic disease using endovascular stent-grafts. Ann Thor Surg 2008; 85:S1-S41 